\title{
THE
}

\section{Fluid Interactions That Enable Stealth Predation by the Upstream- Foraging Hydromedusa Craspedacusta sowerbyi}

K. Lucas

S. P. Colin

J. H. Costello

K. Katja

E. Klos

University of Rhode Island

Follow this and additional works at: https://digitalcommons.uri.edu/gsofacpubs

Terms of Use

All rights reserved under copyright.

\section{Citation/Publisher Attribution}

K. Lucas, S. P. Colin, J. H. Costello, K. Katija, and E. Klos, "Fluid Interactions That Enable Stealth Predation by the Upstream-Foraging Hydromedusa Craspedacusta sowerbyi," The Biological Bulletin 225, no. 1 (August 2013): 60-70. doi: 10.1086/BBLv225n1p60 Available at: https://doi.org/10.1086/BBLv225n1p60

This Article is brought to you for free and open access by the Graduate School of Oceanography at DigitalCommons@URI. It has been accepted for inclusion in Graduate School of Oceanography Faculty Publications by an authorized administrator of DigitalCommons@URI. For more information, please contact digitalcommons-group@uri.edu. 


\title{
Fluid Interactions That Enable Stealth Predation by the Upstream-Foraging Hydromedusa Craspedacusta sowerbyi
}

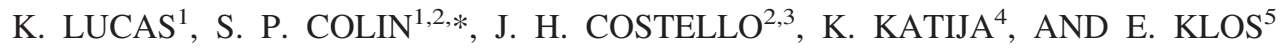 \\ ${ }^{1}$ Biology, Roger Williams University, Bristol, Rhode Island 02809; ${ }^{2}$ Whitman Center, Marine Biological \\ Laboratory, Woods Hole, Massachusetts 02543; ${ }^{3}$ Biology Department, Providence College, Providence, \\ Rhode Island 02918; ${ }^{4}$ Applied Ocean Physics and Engineering, Woods Hole Oceanographic Institution, \\ Woods Hole, Massachusetts 02543; and ${ }^{5}$ Graduate School of Oceanography, University of Rhode
} Island, Narragansett, Rhode Island 02882

\begin{abstract}
Unlike most medusae that forage with tentacles trailing behind their bells, several species forage upstream of their bells using aborally located tentacles. It has been hypothesized that these medusae forage as stealth predators by placing their tentacles in more quiescent regions of flow around their bells. Consequently, they are able to capture highly mobile, sensitive prey. We used digital particle image velocimetry (DPIV) to quantitatively characterize the flow field around Craspedacusta sowerbyi, a freshwater upstream-foraging hydromedusa, to evaluate the mechanics of its stealth predation. We found that fluid velocities were minimal in front and along the sides of the bell where the tentacles are located. As a result, the deformation rates in the regions where the tentacles are located were low, below the threshold rates required to elicit an escape response in several species of copepods. Estimates of their encounter volume rates were examined on the basis of flow past the tentacles, and trade-offs associated with tentacle characteristics were evaluated.
\end{abstract}

\section{Introduction}

Hydromedusae exhibit a broad morphological and functional diversity that enables them to occupy a variety of trophic roles (Mills, 1981; Colin and Costello, 2002; Colin et al., 2003, 2006). Often acting as dominant predators in

Received 15 January 2013; accepted 17 May 2013.

* To whom correspondence should be addressed. E-mail: scolin@ rwu.edu

Abbreviations: EVR, encounter volume rate; DPIV, digital particle imaging velocimetrty. coastal ecosystems, hydromedusae substantially affect zooplankton prey populations (Larson, 1987; Purcell and Grover, 1990; Matsakis and Conover, 1991; Purcell, 2003; Jankowski et al., 2005). Understanding the factors underlying foraging can provide insight into the trophic impact of hydromedusae. Because propulsive mode, swimming performance, bell morphology, and prey selection are all thought to be directly linked to foraging behavior (Costello et al., 2008), analysis of these features ultimately fosters an understanding of the ecological role.

Feeding rates and predatory impact of zooplanktivores are determined by the rate at which prey are encountered and retained. For most pelagic predators, predation is a function of encounter volume rate (EVR), defined as the volume of water the predator can search per unit time (Gerritsen and Strickler, 1977; Kiørboe, 2008, 2011; Colin et al., 2010). The manner in which medusae interact with their surrounding fluid affects their EVR and, therefore, their predation, because medusa feeding and swimming are interrelated processes (Mills, 1981; Daniel, 1983, 1985; Costello, 1992; Costello and Colin, 1994, 1995; Sullivan et al., 1994; Ford et al., 1997; D'ambra et al., 2001). As a result, propulsive modes can be used to broadly describe feeding types, and wake structures have been used to categorize species of medusae into two propulsive modes: jetting and rowing (Colin and Costello, 2002).

Jetting medusae are characterized by having small, prolate bells (fineness ratio $>0.5$ (defined as bell length/bell width)) and constricted orifices leading to the subumbrellar cavity (Daniel, 1983, 1985; Colin and Costello, 2002; Dabiri et al., 2006). Swimming is characterized by rapid, 


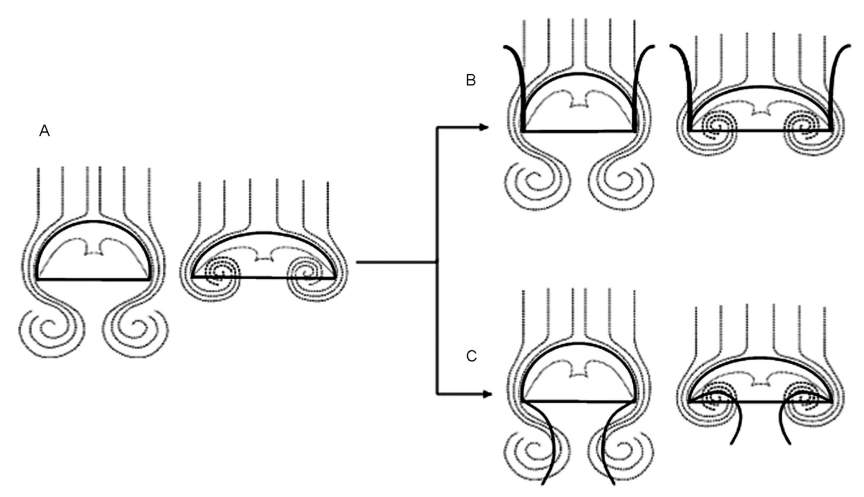

Figure 1. Schematic showing the typical flow field for rowing medusae (A) and how the flow differs around aboral tentacles (B) and oral tentacles (C), demonstrating that flow around the whole bell does not change with tentacle posture. Modified from Colin et al. (2006).

full-body contractions of the bell that propel jets of fluid from the subumbrellar cavity (Daniel, 1983, 1985; Colin and Costello, 2002; Dabiri et al., 2006). This mechanism maximizes thrust (Daniel, 1985; Dabiri et al., 2006), but these medusae are constrained by size, since force required for jet propulsion increases more rapidly than available physiological force as bell diameter increases (Dabiri et al., 2007; Costello et al., 2008). The jet production has a high energy cost (Daniel, 1983, 1985; Dabiri et al., 2006), so jetting medusae drift most of the time, foraging as ambush predators (Colin et al., 2003; Hansson and Kiørboe, 2006).

In contrast, rowing medusae have oblate bells (fineness ratio $<0.5$ ) and are not constrained by size (Dabiri et al., 2007). In these medusae, the bell margin acts as a paddle during bell pulsations, producing starting and stopping vortices rotating in opposite directions relative to one another that are shed from alongside the bell (Fig. 1a; Dabiri et al., 2005). Thrust is generated by the interaction of these vortices, providing a slower but more energy-efficient mode of swimming (Dabiri et al., 2005, 2007, 2010; Sahin et al., 2009). Additionally, this process produces a long, trailing vortex ring wake structure, which entrains large volumes of water behind the bell during both contraction and expansion phases of the pulse cycle (Dabiri et al., 2005). This is advantageous in drawing prey onto capture surfaces located in this region of maximum flow throughout the entire duration of the pulse (Larson, 1991; Dabiri et al., 2005). Few studies have quantified the fluid dynamics associated with prey capture by these rowing medusae (Katija et al., 2011); however, it has been shown that the generation of a feeding current by rowing medusae is energetically efficient (Dabiri et al., 2010) and effective at circulating large volumes through trailing capture surfaces (Dabiri et al., 2005). These traits enable rowing medusae to forage continuously as feeding-current feeders (Colin and Costello, 2002; Colin et al., 2003; Kiørboe, 2011).

To feed, predators must accompany an encounter with a successful capture, in which they strike and retain prey before the prey escape. Tentacles are used by medusae for prey capture, and accordingly, tentacle morphology (number, thickness, and posture) correlates to foraging behavior and prey selection (Mills, 1981; Madin, 1988; Costello and Colin, 1995; Raskoff, 2002; Colin et al., 2006). For example, several lineages of rowing medusae forage with their tentacles positioned aborally (Fig. 1), in front of the bell (termed upstream foragers). Although their tentacle placement differs from that of typical rowing species with orally positioned trailing tentacles, these upstream foragers maintain the same surrounding flow structure as typical rowing medusae (Fig. 1) (Colin et al., 2006). This upstream tentacle posture removes capture surfaces from the region where the trailing vortex rings circulate and where flow is characterized by high flow velocities and high shear deformation rates (Colin et al., 2006). This is important because many zooplankton prey detect fluid disturbances, such as the high-circulation region behind a rowing medusa, and respond with an escape reaction (Kiørboe et al., 1999; Kiørboe and Visser, 1999; Suchman, 2000). Fluid upstream of a rowing medusa's bell, however, is less disturbed (Raskoff, 2002; Colin et al., 2006; Sørnes et al., 2008). It has been suggested that positioning tentacles aborally enables upstream foragers to act as stealth predators (Raskoff, 2002; Colin et al., 2006; Sørnes et al., 2008). Although considerable data document the hydrodynamics and prey selection of downstream-foraging medusae (Costello and Colin, 1994, 1995; Sullivan et al., 1994; Ford et al., 1997; D'ambra et al., 2001; Colin and Costello, 2002; Colin et al., 2003), the fluid interactions of upstream foragers have not been examined quantitatively.

Our study addresses this issue by examining the fluid interactions in Craspedacusta sowerbyi Lankester 1880, a freshwater, upstream-foraging, rowing hydromedusa (Hydrozoa: Olindiidae). Originating in the Yangtze river system in China, C. sowerbyi has been introduced into small lakes, ponds, and water-filled quarries in North America, Europe, and Asia (Kramp, 1951), where it is known to prey on motile, sensitive zooplankton such as rotifers, copepods, and cladocerans in the 0.2-2-mm size range (Dodson and Cooper, 1983; Spadinger and Maier, 1999; Jankowski et al., 2005). Here, we quantify morphological characteristics, fluid flows around swimming individuals, and estimates of encounter volume rate to evaluate the mechanics of $C$. sowerbyi's stealth predation.

\section{Materials and Methods}

\section{Video recording}

Individual adult hydromedusae $(n=4$, diameter $=$ $13.5 \pm 0.5 \mathrm{~mm}( \pm \mathrm{SD})$ ) were hand-collected in jars by scuba divers and immediately transported to the laboratory for analysis. Digital photographs and video recordings 
(SVHS) were made for tentacle counts using white lighting following the methods of Costello and Colin (1994) and Colin and Costello (2002). Spatial characteristics of the optical field were periodically determined from scale bars included in the original recordings. Morphological measurements such as bell diameter and tentacle length were made directly from recordings calibrated with scale bars.

Quantitative flow was measured in the laboratory by using 2D digital particle image velocimetry (DPIV) following the methods of Colin et al. (2010). Individual medusae were placed into filtered seawater seeded with $10-\mu \mathrm{m}$ hollow glass beads. Seeding density was high enough that each interrogation box $(16 \times 16$ pixels $)$ contained more than three particles. Medusae were illuminated through the center of the animal body with a laser sheet (680-nm wavelength). Alignment of the laser light sheet was identified when the manubrium was fully illuminated, and only sequences where the medusa swam perpendicular to the screen were analyzed to ensure that there was no motion in the unmeasured third dimension. Video was recorded at 500 frames s ${ }^{-1}$ using a high-speed digital video camera (Fastcam 1024 PCI; Photron) placed perpendicular to the light sheet. The velocity of an illuminated particle was determined from sequential images analyzed by a cross-correlation algorithm (DaVis 7.2 software by LaVision Inc.). This analysis generated velocity vector fields around the medusa.

For further analysis, each individual's fluid interactions were characterized at six instances throughout the pulsation cycle, where the bell in the first instance in the series was fully relaxed and in the fourth was fully contracted. The second and third instances corresponded to two times equally spaced between the relaxed and contracted states; similarly, the fifth and sixth instances corresponded to two times equally spaced between the contracted state and relaxed state marking the beginning of the next pulse cycle.

\section{Morphological analysis}

Total tentacle count was based on tentacle counts reported in the literature (Mayer, 1910; Payne, 1924; Boulenger and Flower, 1928; Fantham and Porter, 1938; Kramp, 1951; Russell, 1953; Pennak, 1956). Reported total tentacle counts were related to medusa diameter via linear regression, and the mean diameter of the DPIV medusae $(13.5 \pm 0.5 \mathrm{~mm}( \pm \mathrm{SD}))$ was plugged into the resulting equation to yield an estimated total tentacle count (302 tentacles). We classified tentacles into three groups-long, medium, and short—on the basis of length and density (Mayer, 1910). Several still images from the VHS recordings in which the medusa's aboral surface was oriented toward the camera were selected for a count of tentacles in the medium size category. The mean of these counts provided an estimate of the number of medium-length tentacles (40 tentacles, based on three replicates). The long tentacle
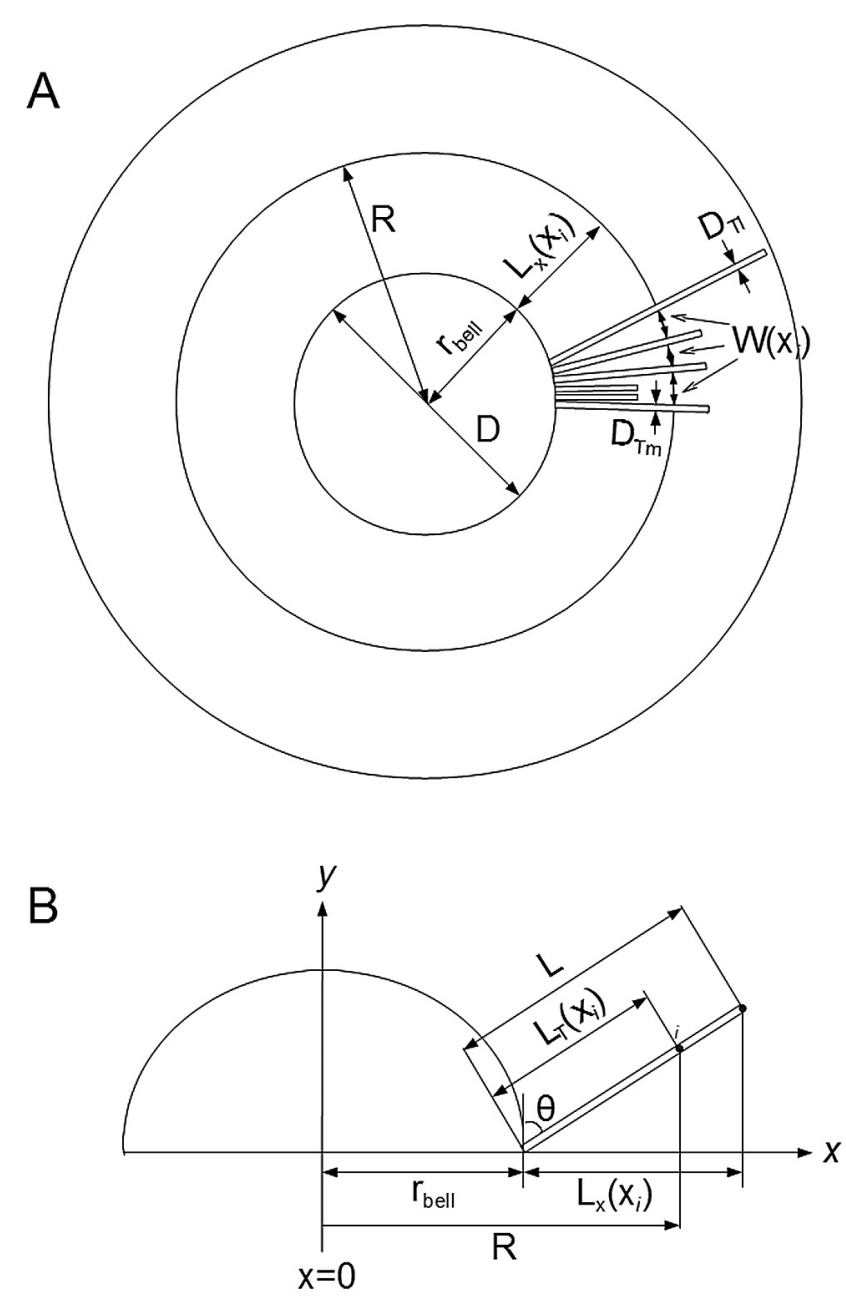

Figure 2. Schematic diagram of a medusa viewed from above (A) and in cross-section (B) showing parameters used in gap width calculations.

category contained only the four perradial tentacles (Kramp, 1951). The number of short tentacles fringing the bell was the difference between the total count and the sum of the number of medium and long tentacles (258 tentacles). Because all of the medusae we observed were of a small size range, these estimates of tentacle counts were adequate for all individuals.

Using the DPIV video sequences, tentacles on one side of the medusa were digitized as series of points using ImageJ 1.44 (developed by the NIH) software. Tentacle length was measured by summing the distances between successive points. Angle from vertical $(\theta)$ was also measured using this software.

Gap width between tentacles (Fig. 2) was calculated as a fraction of the circumference of a circle obtained by looking down on the bell. This circle had a radius $R$, given by

$$
R=r_{\text {bell }}+L_{x}\left(x_{i}\right)
$$

(Equation 1)

where $r_{\text {bell }}$ was the bell radius and $L_{x}\left(\mathrm{x}_{i}\right)$ was the length 
along a tentacle's projection onto the $x$-plane at position $i$ along the tentacle, such that

$$
L_{x}\left(x_{i}\right)=L_{T, i} \sin \theta_{m}
$$

where $L_{T, i}$ was the distance from the bell to position $i$ measured along the tentacle and $\theta_{m}$ was the angle of the medium tentacles from vertical. To account for tentacle thickness, the diameter of a medium-length tentacle $\left(D_{T m}\right)$ was subtracted. Thus, the gap width $(W)$ was calculated as

$$
W=\frac{1}{N}(\pi D)-D_{T m} \quad(\text { Equation 3) }
$$

where $N$ was the number of tentacles of length $L>L_{T, i}$.

Maximum area of tentacle sweep $(S)$ was found using the surface of revolution formula for a function $f(x)$ (Larson et al., 2007),

$$
S=2 \pi \int_{x_{i-1}}^{x_{i}} r(x) \sqrt{1+\left(f^{\prime}(x)\right)^{2}} d x
$$

(Equation 4)

The tentacle was approximated as a series of lines connecting the successive points with $x$-coordinates $x_{i}$ and $x_{i+1}$, so

$$
f^{\prime}(x)=m \quad \text { (Equation 5) }
$$

where $m$ was the slope of the line, and since the axis of rotation was the vertical axis through the bell's apex, a position defined as $x=0, r(x)$, the distance between $f(x)$ and the axis of rotation, was

$$
r(x)=x
$$

(Equation 6)

Substituting and solving the integral led to the formula describing the maximum area swept by the tentacle array:

$$
S=\sum_{i=2}^{n} \pi \sqrt{1=m^{2}}\left(x_{i}^{2}-x_{i-1}^{2}\right)
$$

(Equation 7)

\section{Kinematic analysis}

Frames were extracted from the DPIV video sequences at 0.02 -s time intervals $(t)$, and medusa motion was measured from sequential changes in position $(x, y)$ of the anteriormost point of the exumbrellar surface. Bell velocity $\left(U_{\text {bell }}\right)$ for each time interval was calculated as

$$
U_{\text {bell }}=\frac{\sqrt{\left(x_{n+1}-x_{n}\right)^{2}+\left(x_{n+1}+y_{n}\right)^{2}}}{2}
$$

(Equation 8)

The relative velocity $\left(u_{i}\right)$ between water moving across the tentacle and the medusa at point $i$ along the length of the tentacle was calculated as

$$
u_{i}=u_{w, y, i}-U_{\text {bell }} \sin \theta
$$

where $u_{w, y, i}$ was the component of water velocity (obtained from the DPIV output) in a direction perpendicular to the tentacle at point $i$. Because the medusae were positioned such that the tentacles were not directly aligned with the laser sheet, this calculation resulted in free-stream relative fluid velocities between adjacent tentacles rather than velocities in the tentacle boundary layer.

Localized Reynolds number $\left(R e_{D t, i}\right)$ at position i along the length of the tentacles was approximated using the formula

$$
\operatorname{Re}_{D t, i}=\frac{D_{T}\left|u_{i}\right|}{v}
$$

(Equation 10)

where $\nu$ was the kinematic viscosity of freshwater, and $D_{T}$ was the tentacle diameter $\left(D_{T m}\right.$ was used until position $i$ along tentacle exceeded the length of a medium tentacle, after which $D_{T l}$, the diameter of a long tentacle, was used) (Denny, 1993; Vogel, 1994; Colin and Costello, 2002).

$R e_{D t, i}$ was then used to estimate boundary layer thickness ( $\delta i)$ along the length of the tentacles using the formula

$$
\delta_{i}=0.8 \frac{D_{T}}{\sqrt{R e_{D t, i}}}
$$

(Equation 11)

where, as before, $D_{T m}$ was used until position $i$ along the tentacle exceeded the length of a medium tentacle, after which $D_{T l}$ was used (Feitl et al., 2009). This formula assumes the flow is developed and in steady state.

The velocity vector fields generated in the DPIV analysis enabled measurement of the four components of 2D shear deformation rates (rate of strain) $E_{x x}, E_{x y}, E_{y x}, E_{y y}$ in the flow field, calculated with the equation set below

$$
\begin{aligned}
& E_{x x}=\frac{d u_{x}}{d x} \\
& E_{x y}=\frac{d u_{y}}{d x} \\
& E_{y x}=\frac{d u_{x}}{d y} \\
& E_{y y}=\frac{d u_{y}}{d y}
\end{aligned}
$$

(Equation 12)

where $u$ was the measured planar velocity field. The maximum of these components was used to represent the maximum deformation rate (Colin et al., 2010), since a copepod prey item elicits an escape response when the deformation rate is greater than its threshold regardless of its direction (Kiørboe et al., 1999; Kiørboe and Visser, 1999). 

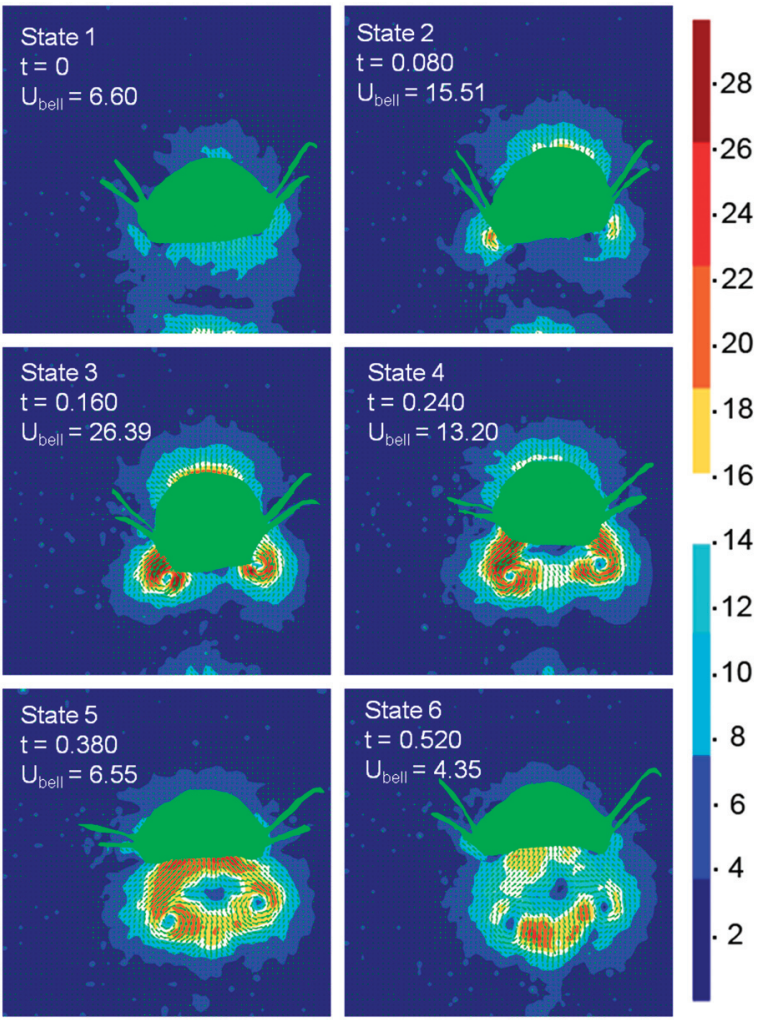

Figure 3. Contours of absolute fluid velocity magnitude for six states ( $t=$ time in seconds; $U_{\text {bell }}=$ bell velocity in $\mathrm{mm} \mathrm{s}^{-1}$ ) representing an entire pulse cycle for a single representative individual (diameter $=13.0 \mathrm{~mm}$ ), with an image of the medusa overlaid. Full pulse was $0.720 \mathrm{~s}$. Values from the right side of the bell only were used in further analysis to eliminate effects from the shadow from the DPIV laser on the left. Green velocity vectors illustrate the direction of the flow (also see Supplementary video [http://www.biolbull.org/content/supplemental/] containing only velocity vectors)

\section{Results}

\section{Flow field around swimming medusae}

Bell pulsations generated a flow field characteristic of rowing medusae, with fluid accelerating orally and becoming entrained in a trailing vortex-ring wake structure (Fig. 3 ). The velocity of fluid surrounding the medusa varied spatially around the bell. Upstream of the medusa, the velocity of the fluid was low relative to the flow downstream throughout the swimming cycle. In the region directly alongside the aboral face, upstream fluid velocities increased, approaching $10 \mathrm{~mm} \mathrm{~s}^{-1}$, during states of maximum contraction when the medusae's swimming velocity peaked (around $20 \mathrm{~mm} \mathrm{~s}^{-1}$ with $R e_{\text {Bell }}=265$ ), but remained lower than fluid velocities downstream of the medusa, where the velocity was in the excess of $25 \mathrm{~mm} \mathrm{~s}^{-1}$ in the vortex-ring wake structure (Fig. 3; see video supplement, http://www.biolbull.edu/content/supplemental). Throughout the pulsation cycle, the lowest fluid velocities around the medusae were observed in the region alongside the bell containing the tentacles $\left(R e_{\text {Tentacles }}<2\right)$; additionally, this region showed minimal velocity gradients (Fig. 3). Peak fluid velocity occurred in the downstream wake region during maximum contraction $(t=0.16$; Fig. 3 ).

In a similar manner, the magnitude of the maximum directional component of the shear deformation rate (rate of strain) varied spatially around the bell. Upstream of the bell, the maximum directional component was low, exceeding about 3-4 s $\mathrm{s}^{-1}$ only in regions directly alongside the aboral surface of the bell during times approaching maximum contraction (Figs. 4, 5). This corresponded to the observed increases in fluid velocities (Fig. 3). The lowest deformation rates around the medusae occurred alongside the bell, in the region containing the tentacles. With few exceptions (where deformation rate approached $5 \mathrm{~s}^{-1}$ near the distal end of the tentacle), the deformation rates along the tentacles were $<3$ $\mathrm{s}^{-1}$ (Figs. 4, 5). In contrast, maximum deformation rates, $>10 \mathrm{~s}^{-1}$ and peaking near $30 \mathrm{~s}^{-1}$, occurred downstream of the bell, in the vortex-ring wake structure (Figs. 4, 5).

\section{Tentacle kinematics}

The profiles of the fluid velocity relative to the tentacles maintained a characteristic shape throughout the pulse cycle but varied in the velocity magnitudes associated with each profile. The relative velocity $\left(u_{i}\right)$ of fluid crossing the proximal end of the tentacle approached $0 \mathrm{~mm} \mathrm{~s}^{-1}$ (Fig. 6). This demonstrated that fluid in the bell's boundary layer translated negligibly relative to the tentacle. Velocities across the tentacles increased away from the bell and remained constantly high beyond the length of the short set of tentacles. In fact, fluid moved across the tentacles throughout the pulse but peaked when the medusae's swimming velocity peaked. The existence of this flow field allowed for boundary layer development on the tentacles.

The boundary layer of the bell could be seen in the low relative velocities near the bell and extended to the tips of the short tentacles. As a result of the low flow over the short tentacles and the close proximity of adjacent tentacles, flow was greatly constricted between the short tentacles. To examine if flow passes between the short tentacles, we compared the boundary layer thickness around the short tentacles to the gap width between tentacles. The smallest gaps between tentacles occurred directly alongside the bell, within the length of the tentacles in the short-length class (Fig. 7). Within this region, the thickness of the boundary layer around the tentacles was often greater than the gap width between tentacles, except during parts of the pulse where flow peaked (Fig. 7 states 2-4). During peak flow, the boundary layer (defined as the region from the no-slip condition to $99 \%$ free stream flow) was about the same size as the gaps, so flow would be constricted during these times, but some fluid would pass between the smallest tentacles. 

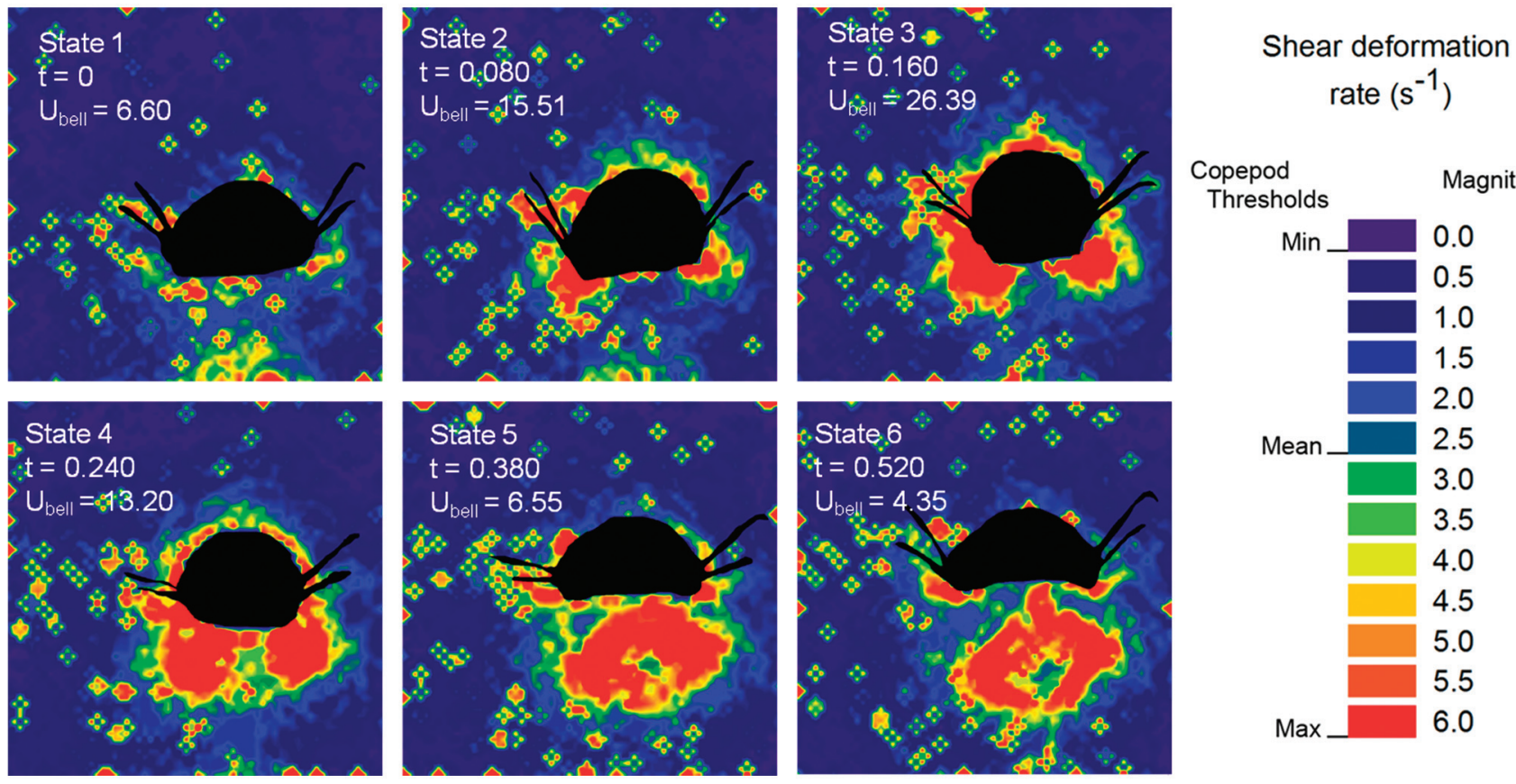

Figure 4. Contours of deformation rate (rate of strain) for six states ( $t=$ time in seconds; $U_{\text {bell }}=$ bell velocity in $\mathrm{mm} \mathrm{s}^{-1}$ ) representing an entire pulse cycle for a representative individual (diameter $=13.0 \mathrm{~mm}$ ), with an image of the medusa overlaid. Full pulse was $0.720 \mathrm{~s}$. Values from the right side of the bell only were used in further analysis, as the shadow from the DPIV laser on the left generated falsely large deformation rate values. Cross-shaped, pixilated patches of concentrated high deformation rates distributed randomly throughout the strain contours represented an artifact from the DaVis software's calculations. Copepod thresholds are adapted from the information in Table 1. Same sequence as shown in Fig. 3.

During other times in the pulse, the boundary layer thickness was greater than the gap width, and little-to-no fluid passed between the tentacles (Fig. 7).

Beyond the length of the short-class tentacles, gap width between tentacles far exceeded the thickness of boundary layers on tentacles (Fig. 7), indicating that encountered fluid could pass freely between tentacles in this region. These results were consistent with the relative velocity profiles on the tentacles, which showed that fluid motion relative to the bell was minimal along the length of the short tentacles (Fig. 7 ), as well as with observations of particle motions in the DPIV video sequences.

\section{Discussion}

\section{Flow and prey detection}

To successfully capture fast, motile zooplankton prey, stealth predators approach their prey with minimal fluid disturbances. Unlike medusae with trailing tentacles whose capture surfaces are located in highly disturbed flow, the tentacles of Craspedacusta sowerbyi are positioned upstream of the bell in a region characterized by low fluid velocities. Therefore, it has been suggested that $C$. sowerbyi and other medusae with tentacles positioned upstream forage as stealth predators. Quantitative analysis of the flow around swimming individuals of $C$. sowerbyi confirms that the aborally positioned tentacles are in regions where deformation rates of the fluid are below the documented response thresholds of copepods (Figs. 4, 5; Table 1). As a result, it is expected that $C$. sowerbyi and other upstream foragers are capable of approaching copepods and other sensitive zooplankton without detection, which confirms previous speculation (Raskoff, 2002; Colin et al., 2006; Sørnes et al., 2008).

The idea that $C$. sowerbyi uses stealth predation to capture prey is also supported by prior laboratory incubation studies in which the species has been shown to readily consume copepods along with less reactive, slower prey such as rotifers and cladocerans (Dodson and Cooper, 1983; Spadinger and Maier, 1999; Jankowski et al., 2005). However, prey size versus clearance rate data presented by Dodson and Cooper (1983) demonstrate a trade-off faced by medusae that forage as upstream predators. Those data showed that clearance rates on slow, less reactive prey such as rotifers and cladocerans increased with prey size, whereas clearance rates on fast, reactive copepods decreased with prey size (Dodson and Cooper, 1983). This suggests that encounter rates limit ingestion of less reactive prey, while capture efficiencies limit ingestion on highly reactive prey. 

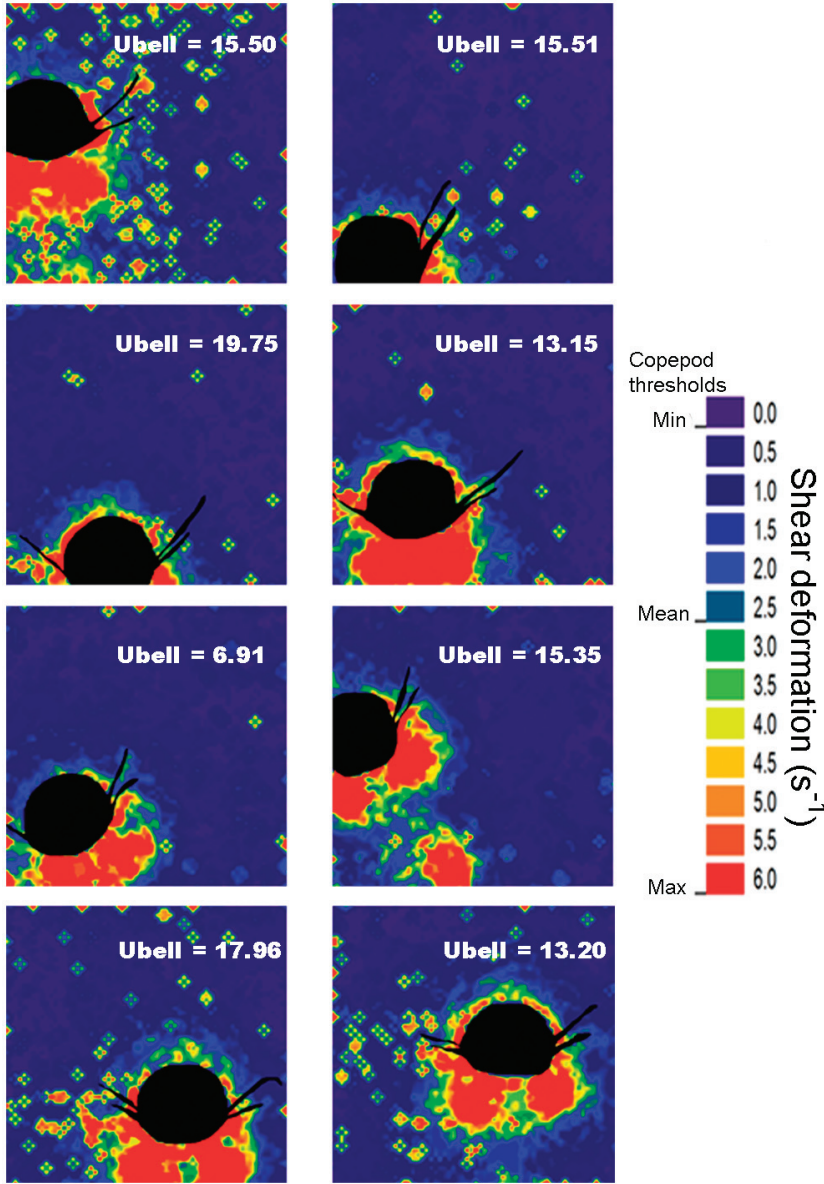

Figure 5. Contours of deformation rate for State 4 for all individuals $(n=4$; mean diameter $=13.5 \mathrm{~mm})$, with an image of the medusa overlaid $\left(U_{\text {bell }}=\right.$ bell velocity in $\left.\mathrm{mm} \mathrm{s}^{-1}\right)$; replicate sequences for each individual are positioned in the same row. Peak deformation rates upstream of the bell occurred during this state. Values from the right side of the bell only were used in further analysis, since the shadow from the DPIV laser on the left generated falsely large values. Cross-shaped, pixilated patches of concentrated high deformation rates represented an artifact from the DaVis software's calculations. Copepod thresholds are adapted from the information in Table 1.

For upstream-foraging medusae, the tentacle array primarily determines encounter rates (Madin, 1988) and capture efficiencies (Colin et al., 2006). The number of upstream tentacles should be directly related to encounter rates with less reactive prey since this increases capture surfaces and decreases spacing between tentacles. However, as tentacle density increases so would the fluid disturbances upstream of the medusae due to the boundary layer and drag of the tentacles. Copepods, the most sensitive prey type of $C$. sowerbyi, detect shear deformation rates in their surrounding fluid to sense predators. If deformation rates are greater than a detection threshold, copepods will react with an escape response and jump away from the predator (Kiørboe et al., 1999; Kiørboe and Visser, 1999). The fluid disturbances generated by the upstream tentacles of $C$. sowerbyi
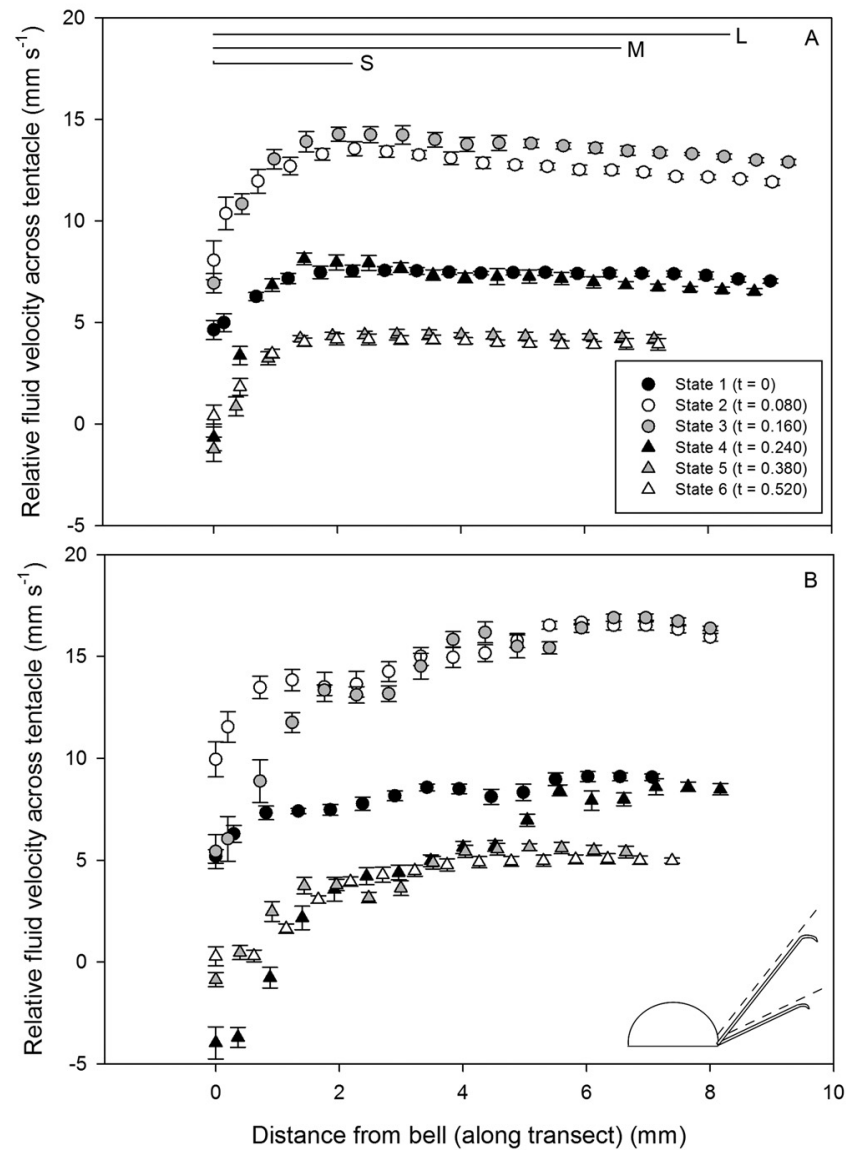

Figure 6. Mean values of relative velocity $\left(u_{i}\right)$ on transects oriented along a representative individual $($ diameter $=13.0 \mathrm{~mm})$ medusa's long $(\mathrm{A})$ and medium (B) tentacles. Schematic shows posture of medium and long tentacles, and dotted lines indicate transects along which velocity profiles were measured. Relative velocity is between the tentacles and the surrounding fluid for a representative individual during 6 states representing an entire pulse cycle. Error bars indicate standard deviation of fluid velocities during 10 successive frames. Full pulse was $0.720 \mathrm{~s}$. Bars labeled $\mathrm{L}, \mathrm{M}$, and $\mathrm{S}$ represent the length of the long, medium, and short tentacles, respectively. Points beyond the length of the bars represent free-stream fluid velocities. Lower velocities within the length of the short tentacles indicate the bell's boundary layer.

were below the average detection limits of copepods whose thresholds have been measured (Figs. 4, 5; Table 1). However, shear deformation rates were above copepod detection limits along the boundary layer of the bell surface and regions with high tentacle densities - that is, the small set of tentacles. High deformation rates in regions with dense tentacle assemblages support the expectation that too many tentacles may reduce the ability of upstream foragers to encounter copepods undetected. Consequently, the observed tentacle arrays of upstream foragers are likely the result of a trade-off between high tentacle densities that maximize prey encounter rates and low tentacle densities that minimize detection by prey. 


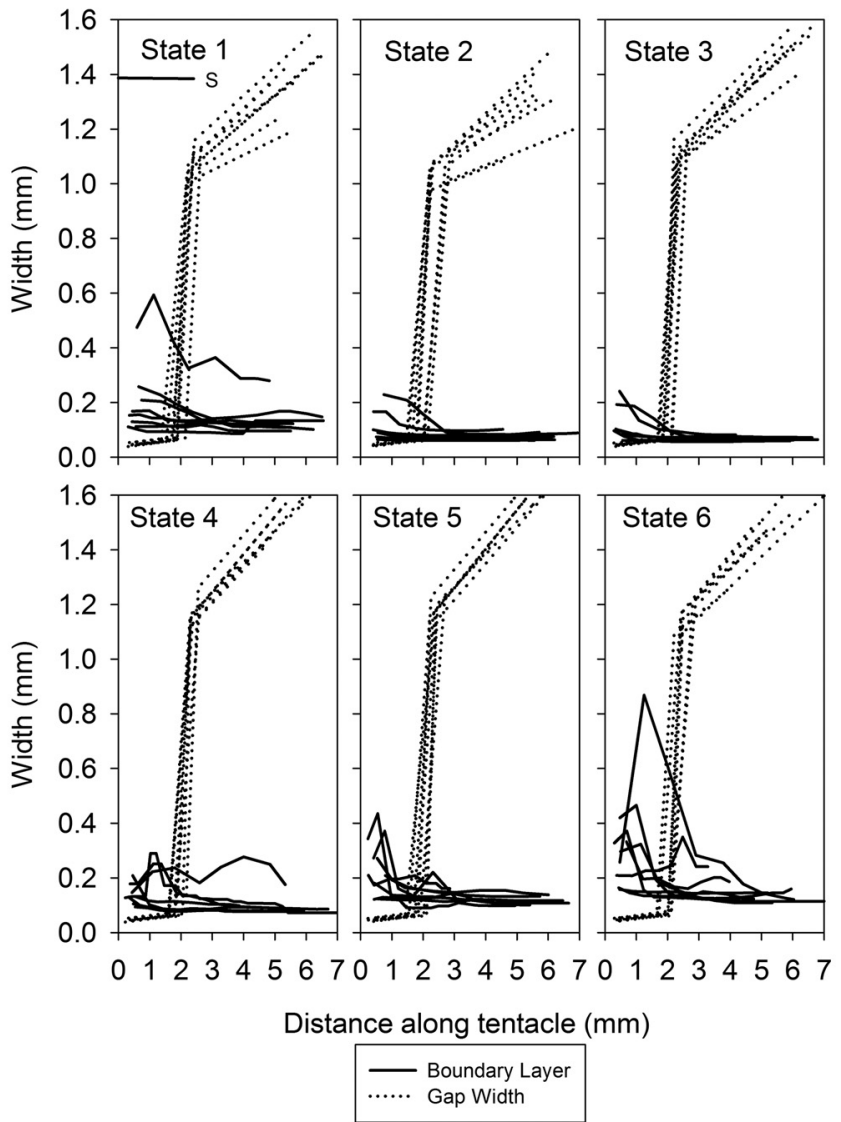

Figure 7. Boundary layer thickness and gap width between tentacles along the length of the medium-sized tentacles. At distances where the solid lines are above the dotted, the boundary layer is thicker than the gap and little fluid is able to pass between the tentacles. At distances where the dotted lines are above the solid, the boundary layer is much smaller than the gaps and fluid is able to pass freely between the tentacles. The six states in sequence represent an entire pulse cycle. Lines represent magnitudes calculated on different medusae $(n=4,2$ replicates per individual; mean diameter $=13.5 \mathrm{~mm}$ ). Line labeled $\mathrm{S}$ illustrates the maximum length of the short tentacles. Long tentacles are excluded to allow better resolution of the boundary layer and gap width thickness on inner tentacles.

\section{Flow and prey encounter}

Fluid flowing through the tentacle array carries planktonic prey and is the basis for prey capture by $C$. sowerbyi. C. sowerbyi possesses three distinct groups of tentacles differing in length, thickness, and abundance. On the basis of our estimates of fluid flows and tentacle dimensions, we expect some fluid to pass between even the smallest, most densely packed tentacles along the bell margin during periods of peak fluid velocities (states 2 and 3; Fig. 6), especially at the tips of these tentacles (Fig. 7 and supplementary video, http://www.biolbull.org/content/supplemental). Video observations confirm that particles were able to pass between these tentacles during limited portions of the swim cycle. We expect that the small-tentacle group likely serves
Table 1

A review of siphon experiments that measured threshold shear deformation rate required for response by several copepod species

\begin{tabular}{|c|c|c|c|}
\hline Species & $\begin{array}{c}\text { Size } \\
(\mathbf{m m})^{*}\end{array}$ & $\begin{array}{l}\text { Threshold } \\
\text { deformation } \\
\text { rate }\left(\mathrm{s}^{-1}\right)\end{array}$ & Source \\
\hline Euchaete rimana & 2.5 & 2.4 & Kiørboe et al., 1999 \\
\hline Pleuromamma xiphias & 5.5 & 4.6 & Kiørboe et al., 1999 \\
\hline Labidocera madurae & 3.3 & 6.3 & Kiørboe et al., 1999 \\
\hline Acartia tonsa, adult & 0.8 & 0.38 & Kiørboe et al., 1999 \\
\hline Acartia tonsa, nauplii & 0.2 & 6.3 & Kiørboe et al., 1999 \\
\hline Acartia tonsa, nauplii & - & 1.4 & Green et al., 2003 \\
\hline Acartia hudsonica & 1.01 & 2.2 & Burdick et al., 2007 \\
\hline Oithona sp. & 0.7 & 3.8 & Kiørboe et al., 1999 \\
\hline Eurytemora affinis & 1 & 1.9 & Kiørboe et al., 1999 \\
\hline $\begin{array}{l}\text { Eurytemora affinis, } \\
\text { nauplii }\end{array}$ & - & 1.0 & Green et al., 2003 \\
\hline Temora longicornis & 1 & 6.5 & Kiørboe et al., 1999 \\
\hline Temora longicornis & 0.74 & 2.7 & Burdick et al., 2007 \\
\hline $\begin{array}{l}\text { Temora longicornis, } \\
\text { nauplii }\end{array}$ & - & 1.5 & Green et al., 2003 \\
\hline Calanus finmarchicus & 0.25 & 0.4 & Kiørboe et al., 1999 \\
\hline Tortanus discaudatus & 1.22 & 0.34 & Burdick et al., 2007 \\
\hline Centropages hamatus & 1.24 & 1.2 & Burdick et al., 2007 \\
\hline
\end{tabular}

* Size refers to copepod prosome length.

to capture primarily small, less responsive zooplankton. In contrast, fluid passed freely between the medium and long groups of tentacles. Consequently, we expect these to be the primary prey capture regions for most of the larger, responsive prey such as copepods.

Although we do not have direct observations of prey retention by $C$. sowerbyi, we can use hydromechanical information to estimate the dominant mechanisms determining prey encounters with the tentacles. Upstream-foraging medusae use their tentacles to capture prey either by sieving the flow, resulting in capture of prey larger than the intertentacle gaps, or by direct interception, whereby small prey (smaller than the inter-tentacle distance) follow streamlines of flow into contact with individual tentacles. The encounter volume rate (EVR) with prey is determined by the volume of fluid that passes through the tentacles over time. For upstream-foraging medusa sieving large prey, this can be approximated from the product of the relative fluid velocity across the tentacles and the conical surface area of the tentacle assemblage (Hansson and Kiørboe, 2006; Kiørboe, 2011). Accordingly, C. sowerbyi's sieving EVR was estimated as $7.11 \pm 2.591 \mathrm{~h}^{-1}$ (Table 2). This estimate would be appropriate for medusae such as Solmissus spp., which forage on gelatinous prey that are frequently larger than the inter-tentacle gaps (Raskoff, 2002; Colin et al., 2006). However, it is not representative of species like Periphylla periphylla (Sørnes et al., 2008) or C. sowerbyi, which forage on crustacean prey that are much smaller than the inter-tentacle gaps. A more appropriate approximation of 
Table 2

Estimated encounter volume rates and clearance rates for Craspedacusta based on calculations from empirical data

\begin{tabular}{|c|c|c|c|}
\hline $\begin{array}{c}\text { Encounter } \\
\text { volume rate* } \\
\left(1 \text { predator }^{-1} \mathbf{h}^{-1}\right)\end{array}$ & Prey & $\begin{array}{l}\text { Clearance rate } \\
\left(1 \text { predator }^{-1} h^{-1}\right)\end{array}$ & Source \\
\hline $1.4 \pm 0.50$ & - & - & Aerosol filtration \\
\hline $7.11 \pm 2.59$ & - & - & $\begin{array}{l}\text { Sieving } \\
\text { Dodson and Cooper }\end{array}$ \\
\hline- & - & 0.04 & $\begin{array}{l}1983 \dagger \\
\text { Spadinger and }\end{array}$ \\
\hline- & - & 0.008 & $\begin{array}{l}\text { Maier, } 1999 \\
\text { Jankowski et al., }\end{array}$ \\
\hline- & Bosmina longirostris & 0.007 & $\begin{array}{l}2005 \\
\text { Jankowski et al., }\end{array}$ \\
\hline- & Other cladocerans & 0.01 & $\begin{array}{l}2005 \\
\text { Jankowski et al., }\end{array}$ \\
\hline- & Nauplii & 0.01 & $\begin{array}{l}2005 \\
\text { Jankowski et al., }\end{array}$ \\
\hline- & Copepodids & 0.008 & $\begin{array}{l}2005 \\
\text { Jankowski et al., }\end{array}$ \\
\hline - & Cyclopoids (adult) & 0.001 & 2005 \\
\hline
\end{tabular}

$* \pm \mathrm{SD} ; n=4,2$ replicates per individual.

$\dagger$ Dodson and Cooper (1983) also report clearance rates up to 2.71 predator ${ }^{-1} \mathrm{~h}^{-1}$ for large nekton killed but not ingested.

EVR for $C$. sowerbyi assumes that the dominant prey encounter mechanism is direct interception and relies upon Re ranging from $0.2-2$ around the tentacles. We can estimate the EVR using intermediate Re estimates (Humphries, 2009) so that

$$
E V R=2 U_{\text {mean }} L_{T} \lambda N \quad \text { (Equation 13) }
$$

where $U_{\text {mean }}$ is the average flow velocity along the tentacle and $\lambda$ is an empirically derived value from Humphries (2009) that is based on prey size (for $C$. sowerbyi, mean prey size is $1.1 \mathrm{~mm}$ ) and describes the width of flow that will cause prey to intercept a cylinder (i.e., tentacle). Based on these calculations, the EVR for $C$. sowerbyi is $1.4 \pm$ $0.50 \mathrm{~h}^{-1}$ (Table 2). This EVR is considerably lower than the EVR estimate for sieving large prey. However, it is considerably higher than reported empirical clearance rates, which were an average of 0.011 predator $^{-1} \mathrm{~h}^{-1}$ (or maximum 0.04; Table 2).

So why is there such a large discrepancy between the theoretical and observed clearance rates? The EVR estimates for direct interception of prey by Craspedacusta sowerbyi represent an upper potential clearance rate that assumes $100 \%$ capture of prey encountered by the medusae. However, post-encounter escape behavior of prey will greatly reduce the percentage of the encountered prey that are actually ingested (termed capture efficiency). High capture efficiencies can occur with some gelatinous predators, such as the ctenophore Mnemiopsis leidyi (Costello et al.,
1999). However, capture efficiencies for upstream-foraging medusae feeding on copepods appear to be low. For example, Colin et al. (2006) reported that the scyphomedusa Nausithoë punctata captured relatively few of the crustacean prey it encountered (adult copepods $=2 \%$ and nauplii $=12 \%$ ). Additionally, small zooplankton may not activate the tentacle's nematocysts (Madin, 1988; Spadinger and Maier, 1999). Consequently, low capture efficiencies can result in clearances rates that are considerably less than theoretical EVRs. Therefore, the discrepancy supports the notion that post-encounter events are most important for determining prey capture for $C$. sowerbyi.

It should also be noted that the theoretical EVR assumes constant swimming, but prior behavioral observations indicate that $C$. sowerbyi spends only about $70 \%$ of its time swimming (Colin et al., 2006). Rather than foraging by continuously swimming, $C$. sowerbyi forages by swimming up the water column, flipping over, and passively sinking back down the water column with tentacles extended (Milne, 1938; Kramp, 1951). If the tentacles are oriented in the sinking direction, they will still serve to encounter prey whether the medusae is actively pulsing or passively sinking because, unlike downstream foragers, bell pulsation is not necessary to transport fluid past the tentacles. For upstream foragers the velocity that the bell moves forward determines the volume of fluid encountered (whether through active swimming or passive sinking). This may be an adaptive advantage of upstream-positioned tentacles. However, the different swimming behaviors entail different flow velocities past the tentacles, and therefore cause deviations from the assumption of continuous swimming that underlies EVR estimates. One pathway to resolution of the differences between potential and realized clearance rates requires determining the details of encounter events as, for example, in Regula et al. (2009), between C. sowerbyi and its prey. Rates of these biological interactions could provide information to modify physically based estimates such as EVR to create a more realistic model of the physical-biological interactions that ultimately determine medusan foraging rates.

\section{Acknowledgments}

We thank Edmund Chaitman and Julia Streit for their generous hospitality and guidance during the collecting trip and the reviewers for valuable comments that improved earlier versions of the manuscript. This research is supported by the National Science Foundation awarded to SPC (OCE- 0623534 and 0727544) and JHC (OCE-0351398 and OCE-0623534) and by the Office of Naval Research awarded to JHC (N000140810654). K. Lucas was partially funded by EPSCoR Cooperative Agreement \#EPS-1004057 to the State of Rhode Island SURF award. S. Colin was also supported by RWU Foundation to Promote Scholarship and Teaching. 


\section{Literature Cited}

Boulenger, C. L., and W. U. Flower. 1928. Craspedacusta sowerbii, and its identity with C. (Microhydra) ryderi. Proc. Zool. Soc. Lond. 98: 1005-1014.

Burdick, D. S., D. K. Hartline, and P. H. Lenz. 2007. Escape strategies in co-occurring calanoid copepods. Limnol. Oceanogr. 52: 2373-2385.

Colin, S. P., and J. H. Costello. 2002. Morphology, swimming performance and propulsive mode of six co-occurring hydromedusae. J. Exp. Biol. 205: 427-437.

Colin, S. P., J. H. Costello, and E. Klos. 2003. In situ swimming and feeding behavior of eight co-occurring hydromedusae. Mar. Ecol. Prog. Ser. 253: 305-309.

Colin, S. P., J. H. Costello, and H. Kordula. 2006. Upstream foraging by medusae. Mar. Ecol. Prog. Ser. 327: 143-155.

Colin, S. P., J. H. Costello, L. J. Hansson, J. Titelman, and J. O. Dabiri. 2010. Stealth predation and the predatory success of the invasive ctenophore Mnemiopsis leidyi. Proc. Natl. Acad. Sci. USA 107: 1722317227.

Costello, J. H. 1992. Foraging mode and energetics of hydrozoan medusae. Sci. Mar. 56: 185-191.

Costello, J. H., and S. P. Colin. 1994. Morphology, fluid motion and predation by the scyphomedusa Aurelia aurita. Mar. Biol. 121: 327334.

Costello, J. H., and S. P. Colin. 1995. Flow and feeding by swimming scyphomedusae. Mar. Biol. 124: 399-406.

Costello, J. H., R. Loftus, and R. Waggett. 1999. Influence of prey detection on capture success for the ctenophore Mnemiopsis leidyi feeding upon adult Acartia tonsa and Oithona colcarva copepods. Mar. Ecol. Prog. Ser. 191: 207-216.

Costello, J. H., S. P. Colin, and J. O. Dabiri. 2008. Medusan morphospace: phylogenetic constraints, biomechanical solutions, and ecological consequences. Invertebr. Biol. 127: 265-290.

Dabiri, J. O., S. P. Colin, J. H. Costello, and M. Gharib. 2005. Flow patterns generated by oblate medusan jellyfish: field measurements and laboratory analyses. J. Exp. Biol. 208: 1257-1265.

Dabiri, J. O., S. P. Colin, and J. H. Costello. 2006. Fast-swimming hydromedusae exploit velar kinematics to form an optimal vortex wake. J. Exp. Biol. 209: 2025-2033.

Dabiri, J. O., S. P. Colin, and J. H. Costello. 2007. Morphological diversity of medusan lineages constrained by animal-fluid interactions. J. Exp. Biol. 210: 1868-1873.

Dabiri, J. O., S. P. Colin, K. Katija, and J. H. Costello. 2010. A wake-based correlate of swimming performance and foraging behavior in seven co-occurring jellyfish species. J. Exp. Biol. 213: 1217-1225.

D'ambra, I., J. H. Costello, and F. Bentivegna. 2001. Flow and prey capture by the scyphomedusa Phyllorhiza punctata von Lendenfeld, 1884. Hydrobiologia 451: 223-227.

Daniel, T. L. 1983. Mechanics and energetics of medusan jet propulsion. Can. J. Zool. 61: 1406-1420.

Daniel, T. L. 1985. Cost of locomotion: unsteady medusan swimming. J. Exp. Biol. 119: 149-164.

Denny, M. 1993. Air and Water: The Biology and Physics of Life's Media. Princeton University Press, Princeton, NJ.

Dodson, S. I., and S. D. Cooper. 1983. Trophic relationships of the freshwater jellyfish Craspedacusta sowerbyi Lankester 1880. Limnol. Oceanogr. 28: 345-351.

Fantham, H. B., and A. Porter. 1938. Occurrence of the freshwater medusa, Craspedacusta sowerbii, in Eastern Canada. Nature 141: $515-516$.

Feitl, K. E., A. F. Millet, S. P. Colin, J. O. Dabiri, and J. H. Costello. 2009. Functional morphology and fluid interactions during early development of the scyphomedusa Aurelia aurita. Biol. Bull. 217: 283-291.

Ford, M. D., J. H. Costello, K. B. Heidelberg, and J. E. Purcell. 1997.
Swimming and feeding by the scyphomedusa Chrysaora quinquecirrha. Mar. Biol. 129: 355-362.

Gerritsen, J., and J. R. Strickler. 1977. Encounter probabilities and community structure in zooplankton: a mathematical model. J. Fish. Res. Board Can. 34: 73-82.

Green, S., A. Visser, J. Titelman, and T. Kiørboe. 2003. Escape responses of copepod nauplii in the flow field of the blue mussel, Mytilus edulis. Mar. Biol. 142: 727-733.

Hansson, L. J., and T. Kiørboe. 2006. Prey-specific encounter rates and handling efficiencies as causes of prey selectivity in ambushfeeding hydromedusae. Limnol. Oceanogr. 51: 1849-1858.

Humphries, S. 2009. Filter feeders and plankton increase particle encounter rates through flow regime control. Proc. Natl. Acad. Sci. USA 106: $7882-7887$.

Jankowski, T., T. Strauss, and H. T. Ratte. 2005. Trophic interactions of the freshwater jellyfish Craspedacusta sowerbii. J. Plankton Res. 27: 811-823.

Katija, K., W. T. Beaulieu, C. Regula, S. P. Colin, J. H. Costello, and J. O. Dabiri. 2011. Quantification of flows generated by the hydromedusa Aequorea victoria: a Lagrangian coherent structure analysis. Mar. Ecol. Prog. Ser. 435: 111-123.

Kiørboe, T. 2008. A Mechanistic Approach to Plankton Ecology. Princeton University Press, Princeton, NJ.

Kiørboe, T. 2011. How zooplankton feed: mechanisms, traits and tradeoffs. Biol. Rev. 86: 311-339.

Kiørboe, T., and A. Visser. 1999. Predator and prey perception in copepods due to hydromechanical signals. Mar. Ecol. Prog. Ser. 179: 81-95.

Kiørboe, T., E. Saiz, and A. Visser. 1999. Hydrodynamic signal perception in the copepod Acartia tonsa. Mar. Ecol. Prog. Ser. 179: 97-111.

Kramp, P. L. 1951. Freshwater medusae in China. Proc. Zool. Soc. Lond. 120: 165-184.

Larson, R., R. Hostetler, and B. H. Edwards. 2007. Calculus of a Single Variable. Houghton Mifflin, Boston, MA.

Larson, R. J. 1987. Trophic ecology of planktonic gelatinous predators in Saanich Inlet, British Columbia: diets and prey selection. J. Plankton Res. 9: 811-820.

Larson, R. J. 1991. Diet, prey selection, and daily ration of Stomolophus meleagris, a filter-feeding scyphomedusa from the NE Gulf of Mexico. Estuar. Coast. Shelf Sci. 32: 511-525.

Madin, L. P. 1988. Feeding behavior of tentaculate predators: in situ observations and a conceptual model. Bull. Mar. Sci. 43: 413-429.

Matsakis, S., and R. J. Conover. 1991. Abundance and feeding of medusae and their potential impact as predators on other zooplankton in Bedford Basin (Nova Scotia, Canada) during spring. Can. J. Fish. Aquat. Sci. 48: 1419-1430.

Mayer, A. G. 1910. Medusae of the World, Vol. 1, The Hydromedusae. Carnegie Institute of Washington, Washington, DC.

Mills, C. E. 1981. Diversity of swimming behaviors in hydromedusae as related to feeding and utilization of space. Mar. Biol. 64: 185-189.

Milne, L. J. 1938. Some aspects of the behavior of the freshwater jellyfish Craspedacusta sp. Am. Nat. 72: 464-472.

Payne, F. 1924. A study of the fresh-water medusa, Craspedacusta ryderi. J. Morphol. 38: 387-411.

Pennak, R. W. 1956. The fresh-water jellyfish Craspedacusta in Colorado with some remarks on its ecology and morphological degeneration. Trans. Am. Microsc. Soc. 75: 324-331.

Purcell, J. E. 2003. Predation on zooplankton by large jellyfish Aurelia labiata, Cyanea capillata, and Aequorea aequorea, in Prince William Sound, Alaska. Mar. Ecol. Prog. Ser. 246: 137-152.

Purcell, J. E., and J. J. Grover. 1990. Predation and food limitation as causes of mortality in larval herring at a spawning ground in British Columbia. Mar. Ecol. Prog. Ser. 59: 55-61. 
Raskoff, K. A. 2002. Foraging, prey capture, and gut contents of the mesopelagic narcomedusa Solmissus spp. (Cnidaria: Hydrozoa). Mar. Biol. 141: 1099-1107.

Regula, C., S. P. Colin, J. H. Costello, and H. Kordula. 2009. Prey selection mechanism of ambush-foraging hydromedusae. Mar. Ecol. Prog. Ser. 374: 135-144.

Russell, F. S. 1953. The Medusae of the British Isles, Vol. 1, Anthomedusae, Leptomedusae, Limnomedusae, Trachymedusae, and Narcomedusae. Cambridge University Press, Cambridge.

Sahin, M., K. Mohseni, and S. P. Colin. 2009. The numerical comparison of flow patterns and propulsive performances for the hydromedusae Sarsia tubulosa and Aequorea victoria. J. Exp. Biol. 212: 2656-2667.

Sørnes, T. A., A. Hosia, U. Båmstedt, and D. L. Aksnes. 2008. Swim- ming and feeding in Periphylla periphylla (Scyphozoa, Coronatae). Mar. Biol. 153: 653-659.

Spadinger, R., and G. Maier. 1999. Prey selection and diel feeding of the freshwater jellyfish, Craspedacusta sowerbyi. Freshw. Biol. 41: 567-573.

Suchman, C. L. 2000. Escape behavior of Acartia hudsonica copepods during interactions with scyphomedusae. J. Plankton Res. 22: $2307-$ 2323.

Sullivan, B. K., J. R. Garcia, and G. Klein-MacPhee. 1994. Prey selection by the scyphomedusan predator Aurelia aurita. Mar. Biol. 121: 335-341.

Vogel, S. 1994. Life in Moving Fluids. Princeton University Press, Princeton, NJ. 